\title{
Active exercise promotes Achilles tendon healing and is accompanied by the upregulation of collapsin response mediator protein-2 in rats
}

\author{
BAYIXIATI QIANMAN*, AYIDAER JIALIHASI*, BATIZA ASILEHAN, ALIYA KUBAI, \\ RAKIMBAIEV AIBEK, AIKEREMU WUPUER, WULANBAI TANGKEJIE, ABUDOUHEILIL MAIMAITIAILI, \\ NUERAI SHAWUTALI, AYNAZ BADELHAN, ADILI AIZEZI, AMUDING AISAIDING, JIANATI WUERLIEBIEKE, \\ YERZAT BAKYT, ELIHAER MAKEMUTIBIEKE and JIASHARETE JIELILE
}

\begin{abstract}
Department of Orthopedics Centre, The First Teaching Hospital of Xinjiang Medical University, Kazakh Medical Association of Xinjiang Uygur Autonomous Region and The Sports Medicine Research Centre of Orthopedics Research Institute, Urumqi, Xinjiang Uygur Autonomous Region 830054, P.R. China
\end{abstract}

Received December 31, 2015; Accepted January 20, 2017

DOI: $10.3892 / \mathrm{mmr} .2017 .6889$

\begin{abstract}
Collapsin response mediator protein-2 (CRMP-2) is involved in neurite elongation and regeneration; however, its role in wound healing remains to be elucidated. The present study aimed to investigate the effects of active mobilization treatment on Achilles tendon healing and to determine the role of CRMP-2 in the healing process. Sprague Dawley rats were subjected to Achilles tendon injury, which was verified by hematoxylin and eosin staining and scanning electronic microscopy. Immobilization induced the disruption of collagen fibril arrangement and promoted collagen fibril damage. The average collagen fibril perimeter in the active mobilization group was significantly increased compared with in the immobilization group $(125.6 \pm 0.8 \mathrm{~nm}$ vs. $119.9 \pm 1.7 \mathrm{~nm}$; $\mathrm{P}<0.05)$. In addition, immunohistological analysis revealed that CRMP-2 expression was significantly upregulated, particularly in the ruptured site of Achilles tendon tissues derived from animals in the mobilization group compared with the immobilization group $(0.32 \pm 0.00$ vs. $0.08 \pm 0.00 ; \mathrm{P}<0.05)$. The increased CRMP-2 levels were also confirmed by western blotting (active mobilization group, 0.71 \pm 0.03 ; immobilization
\end{abstract}

Correspondence to: Dr Jiasharete Jielile, Department of Orthopedics Centre, The First Teaching Hospital of Xinjiang Medical University, Kazakh Medical Association of Xinjiang Uygur Autonomous Region and The Sports Medicine Research Centre of Orthopedics Research Institute, 137 Liyushan Road, Urumqi, Xinjiang Uygur Autonomous Region 830054, P.R. China

E-mail: jingping-bai@163.com

*Contributed equally

Key words: Achilles tendon, active mobilization, immobilization, collagen fibril, collapsin response mediator protein-2 group, $0.49 \pm 0.01 \mathrm{~nm} ; \mathrm{P}<0.05)$. These results indicated that active mobilization may promote Achilles tendon healing via upregulation of CRMP-2 protein expression.

\section{Introduction}

The Achilles tendon is the strongest tendon in the human body. Achilles tendon ruptures frequently occur in sporting activities (1) and patients with Achilles tendon rupture have been reported to have a 200 -fold risk of sustaining a contralateral rupture (2). Surgery has the highest success rate, particularly when dealing with elite athletes; however, it is associated with a significantly high risk of postoperative complications (3). Non-surgical treatment, such as exercise, may be considered a promising approach for Achilles tendon healing. The favorable effect of early functional rehabilitation has been addressed in previous studies (4-7). However, the underlying mechanism involved in active functional rehabilitation-mediated Achilles tendon healing requires further clarification.

The pathology of the Achilles tendon following injury is poorly understood. Collagen fibrils represent the smallest unit in tendons, which aggregate to form fascicles that are responsible for tensile strength (8). In the immediate area of the rupture site, a loss of larger collagen fibrils (9), a more evident gelatinolytic activity and altered matrix metalloprotease (MMP) expression (10) has previously been identified.

Collapsin response mediator protein-2 (CRMP-2), which is a mammalian homologue of UNC-33, is critical for determining the fate of axons and dendrites, and contributes to the establishment and maintenance of neuronal polarity (11). Our previous study identified several differentially expressed proteins in injured Achilles tendon tissue (12). Among these differentially expressed proteins, CRMP-2 expression was upregulated in animals receiving active rehabilitation treatment, suggesting that CRMP-2 may be involved in the process of Achilles tendon healing (12).

The present study established a rat model of Achilles tendon injury and determined the efficacy of active 
mobilization therapy on Achilles tendon healing and collagen fibril arrangement. In addition, the potential involvement of CRMP-2 in mobilization-induced Achilles tendon healing was investigated. The findings suggested that early active exercise may be a potential strategy for rehabilitation therapy and CRMP-2 may be involved in efficient Achilles tendon healing.

\section{Materials and methods}

Animals. A total of 45 healthy, male, 4-month-old, adult Sprague Dawley rats, weighing 230 $\pm 20 \mathrm{~g}$, were obtained from the Laboratory Animal Center, Xinjiang Medical University (Urumqi, China). The rats were housed in specific pathogen-free conditions and had ad libitum access to food and water prior to the experiment. Housing conditions were thermostatically maintained at $25^{\circ} \mathrm{C}$ with a $12 \mathrm{~h}$ light/dark cycle. The Animal Ethical Committee of The First Teaching Hospital of Xinjiang Medical University (Urumqi, China) approved the animal experiments.

Achilles tendon injury model in rats. In order to establish the rodent Achilles tendon injury model, all rats were fasted for $12 \mathrm{~h}$ and deprived of water for $4 \mathrm{~h}$. Surgical procedures were conducted under standard aseptic technique, using a combination of local anesthesia, utilizing $12 \mathrm{ml} 0.25 \%$ procaine hydrochloride injection (Sichuan Kelun Bio-Tech Pharmaceutical Co., Ltd., Chengdu, China) and hypnotic induction (neck massage 45 times per minute) as previously described (13). Briefly, following anesthesia, an S-shaped incision was made on the right Achilles tendon of each rat and the Achilles tendon was carefully freed from the underlying tissue under aseptic conditions. The Achilles tendon injury was then repaired using a previously described surgical procedure (13). The incision was covered with sterile gauze and clean and dry bandage. The animals were returned to their cages postoperatively.

Experimental setup. Rats with Achilles tendon injury were randomly divided into two groups, the immobilization group $(n=15)$ and the active mobilization group $(n=15)$. The rats in the immobilization group had their operated leg immobilized with a fixation brace. The ankle was fixed at a $90^{\circ}$ angle and the knee joint at $85^{\circ}$. Following leg immobilization with a plaster cast, the animals were returned to their cages. The rats in the active mobilization group received training by offering water at a place $13 \mathrm{~cm}$ higher than usual following surgery, in order to increase the rats' active raising and squatting movements. Therefore, post-operation, the average number of raising and squatting movements was increased to $\sim 150 \pm 15$ times/day, to ensure rats continued exercising. All rats were returned to their cages and did not receive additional treatments. Additionally, 15 healthy rats were included for the comparison of structure of Achilles tendon under light microscope.

Inclusion and exclusion criteria. Animals meeting the following criteria post-surgery were included: i) Stage I incision healing (endogenous healing at the site of incision) (14); and ii) stage I tissue healing of the injured Achilles tendon. Animals were excluded if any of the following situations occurred: i) Death; ii) infection at the site of incision; iii) sample contamination; iv) injured Achilles tendon had a gap $>1 \mathrm{~mm}$ in length and v) cast was loose or had fallen off.

Sample collection. All rats were sacrificed 14 days after the operation and the Achilles tendon tissues were carefully removed. Tissue samples were washed 3 times with cold normal saline and longitudinally cut into 2 pieces. One piece was used for histological examination, and the other piece was prepared for western blotting. For histological examination, each sample was cut into 30 slices, and used for hematoxylin and eosin (H\&E) staining (10 slices), immunohistochemical staining (10 slices) and scanning electronic microscopy (SEM) (10 slices). All tissue samples were stored in liquid nitrogen until use.

Histological examination. Tissue samples from the immobilization $(n=13)$, active mobilization groups $(n=12)$, and healthy control $(n=15)$ were fixed in $10 \%$ formalin and subsequently dehydrated in an ethanol series (75, 85, 95 and 100\%). The samples were then cleared in xylene and embedded in paraffin for histological analysis. Paraffin-embedded tissues were sectioned on a microtome to $4 \mu \mathrm{m}$ in an automatic tissue processor (LEICA RM2235; Leica Microsystems, Inc., Buffalo Grove, IL, USA) and were then stained with H\&E. A total of forty microscopic fields were randomly selected and images were captured under light microscopy (magnification, x400; The automated Leica DM3000 B microscope (Leica Microsystems, Inc.).

SEM analysis. Tissue samples from the immobilization $(n=13)$ and the active mobilization group $(n=12)$ were fixed in $3 \%$ glutaraldehyde for $2-4 \mathrm{~h}$ at $4^{\circ} \mathrm{C}$ and post-fixed in $1 \%$ osmium tetroxide solution for a further $2 \mathrm{~h}$. Following dehydration in an ethanol and acetone gradient $[50 \%(\mathrm{v} / \mathrm{v})$ ethanol (10 min), $70 \%$ (v/v) ethanol (10 min), 80\% (v/v) ethanol (10 min), $90 \%$ (v/v) ethanol (10 min), 90\% (v/v) acetone (10 min), 100\% acetone (3x15 min)], samples were impregnated with a mixture of acetone/resin (1:1) with gentle rotation overnight) and incubated with resin at room temperature for a further $1 \mathrm{~h}$. Samples were then sectioned with an LKB 2188 ultramicrotome into semi-thin and ultra-thin sections. The semi-thin sections were stained with toluidine blue and the ultra-thin sections with super saturated uranyl acetate and lead citrate. The ultra-thin sections were examined using scanning electron microscopy (SEM). Images were obtained under a JEOL JEM 1230 SEM (JEOL Ltd., Tokyo, Japan) at magnification x20,000. The ultrastructure of 10 randomly selected collagen fibrils was examined and compared between the experimental groups. The average circumference of each collagen fibril was quantified and averaged as previously described (15).

Immunohistochemical analysis. Paraffin-embedded tissue sections prepared at a size of $25 \times 20 \times 25 \mathrm{~mm}^{3}(\mathrm{n}=13$ for immobilization group; $n=12$ for active mobilization group) were exposed to heating in EDTA buffer ( $\mathrm{pH}$ 8.0) for antigen retrieval and were then treated with endogenous peroxidase (3\% hydrogen peroxide solution) for $5 \mathrm{~min}$ at room temperature. Following blocking in 5\% goat serum (Sigma-Aldrich; Merck KGaA, Darmstadt, Germany) for 30-40 min at room temperature, sections were immunostained with an 
Control

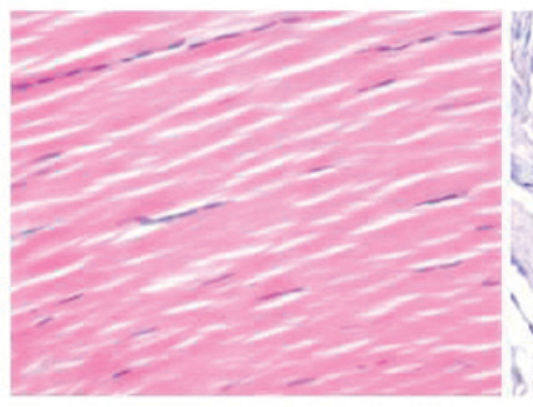

Immobilization

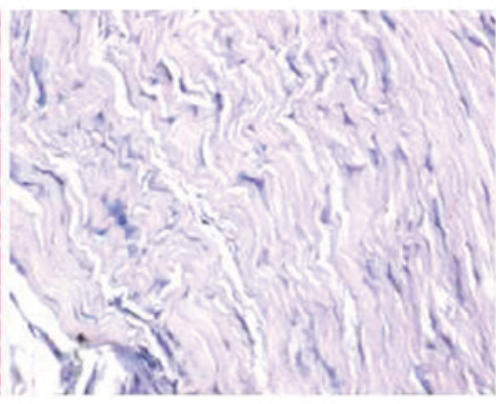

Mobilization

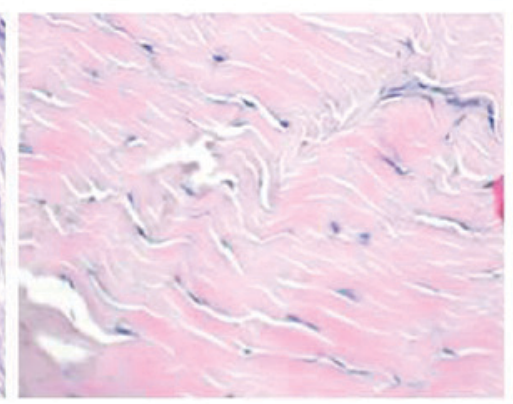

Figure 1. Histological examination of the Achilles tendon tissues derived from the various experimental groups. The Achilles tendon tissue samples derived from healthy control, immobilization, or active mobilization groups were stained with hematoxylin and eosin. Magnification, $\mathrm{x} 400$.

anti-CRMP-2 primary antibody (cat. no. sc-30228; 1:1,500; Santa Cruz Biotechnology, Inc., Dallas, TX, USA) at $37^{\circ} \mathrm{C}$ for $3 \mathrm{~h}$. Subsequently, sections were washed 3 times in PBS, incubated with a biotin-labeled goat anti-rabbit secondary antibody (cat. no. LS-C60867-1000; 1:4,000; LifeSpan BioSciences, Inc., Seattle, WA, USA) for $20 \mathrm{~min}$ at room temperature and incubated with avidin-conjugated horseradish peroxidase (HRP, Sigma-Aldrich; Merck KGaA) at a dilution of 1:100 in 0.1 M PBS for $15 \mathrm{~min}$ at room temperature. The sample was washed 3 times in PBS and visualized using 3, 3'-diaminobenzidine tetrahydrochloride. Subsequently, samples were counterstained with hematoxylin. A total of 5 micrographs were randomly selected from each slice and images were captured using a LEICA DM3000 microscope and a charge-coupled device camera at magnification $\mathrm{x} 400$. The mean optical density (MOD) in each image was analyzed using Image-Pro Plus version 6.0 software (Media Cybernetics, Inc., Rockville, MD, USA), and the mean CRMP-2 intensity in tissue samples was quantified.

Western blotting. A total of 7 samples were randomly selected from each experimental group. Total protein was extracted from tissues using radioimmunoprecipitation assay lysis buffer (Beijing Solarbio Science \& Technology Co., Ltd., Beijing, China). Protein concentration was quantified using bicinchoninic acid assay kit (Thermo Fisher Scientific, Inc., Waltham, MA, USA). Equal amounts of protein $(40 \mu \mathrm{g})$ were then separated by $10 \%$ sodium dodecyl sulfate-polyacrylamide gel electrophoresis and transferred to a polyvinylidene fluoride membrane (0.45 $\mu \mathrm{m}$; Merck Millipore, Darmstadt, Germany). Membranes were blocked with Tris-buffered saline plus $0.1 \%$ Tween-20 (TBST) with 5\% w/v non-fat dry milk for $50 \mathrm{~min}$ at room temperature. Following blocking, the membranes were incubated with anti-CRMP-2 (cat. no. SC-30228; 1:300; Santa Cruz Biotechnology, Inc.) or anti- $\beta$-actin (cat. no. SC-81178; 1:1,000; Santa Cruz Biotechnology, Inc.) primary antibodies at $4^{\circ} \mathrm{C}$ overnight. Membranes were washed 3 times with TBST and incubated with HRP-labeled anti-rabbit secondary antibody (cat. no. LS-C60867-1000; 1:4,000; LifeSpan BioSciences Inc.) for $1 \mathrm{~h}$ at room temperature. Followed by washing with TBST, immunodetected protein bands were visualized using enhanced chemiluminescence kits according to the manufacturer's protocol (Thermo Fisher Scientific, Inc.) and images were captured using a Chemi Doc MP gel imaging system
(Bio-Rad Laboratories, Inc., Hercules, CA, USA). Protein levels were quantified by analyzing the densitometric values using ImageJ software version 1.48 for Windows (National Institutes of Health, Bethesda, MD, USA). The housekeeping protein $\beta$-actin was used as an internal control.

Statistical analysis. Data are presented as the mean and standard deviation of at least 3 independent experiments and were analyzed using SPSS version 20.0 software (IBM SPSS, Armonk, NY, USA). Data with normal distribution was tested for homogeneity of variance. Statistical significance was determined using a Student's t-test (Independent Samples) for equal variances and Welch's t-test was used for the unequal variances with a $95 \%$ confidence interval. $\mathrm{P}<0.05$ was considered to indicate a statistically significant difference.

\section{Results}

Histological examination. Rats were randomly divided into immobilization $(n=15)$ and active mobilization groups $(n=15)$. A total of 5 animals were excluded according to the aforementioned criteria. In the immobilization group, one animal had an infection at the site of incision and the cast fell off another animal. In the active mobilization group, 3 animals were excluded for the following reasons: Death $(n=1)$, infection at the site of incision $(n=1)$ and injured Achilles tendon had a gap $>1 \mathrm{~mm}$ in length $(\mathrm{n}=1)$. Therefore, the immobilization group contained 13 rats and the active mobilization group contained 12 rats that were used for subsequent analyses. Histological methods were used to compare the various pathological manifestations following Achilles tendon injury. In healthy animals, the structure of the Achilles tendon was intact, with collagen fibrils, stained in pink, arranged in regular, tightly lined and evenly arranged parallel arrays with abundant capillaries (Fig. 1). Achilles tendon tissue samples derived from the immobilization group were markedly different in appearance. They exhibited a loss of collagen fibrils, disturbed collagen fibril arrangement, a reduced number of fibroblasts, formation of thin-walled capillaries, formation of granulation tissue at the site of injury and infiltration of inflammatory cells (Fig. 1). Notably, in the active mobilization rehabilitation therapy group these pathological manifestations were reduced. Animals in the mobilization group exhibited regular collagen fibril arrangement, increased numbers of collagen fibrils, 

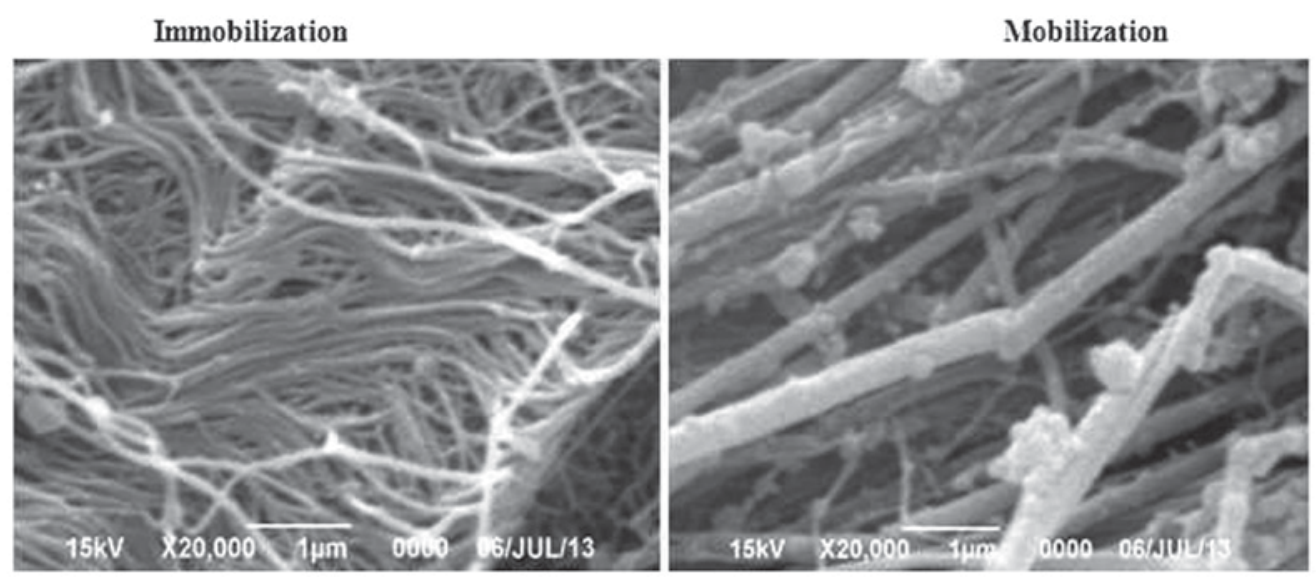

Figure 2. Ultrastructural alterations in Achilles tendon tissues. The ultrastructure of the Achilles tendon tissues was observed in rats from the immobilization and active mobilization groups by scanning electron microscopy. Magnification, x20,000. Scale bar, $1 \mu \mathrm{m}$.

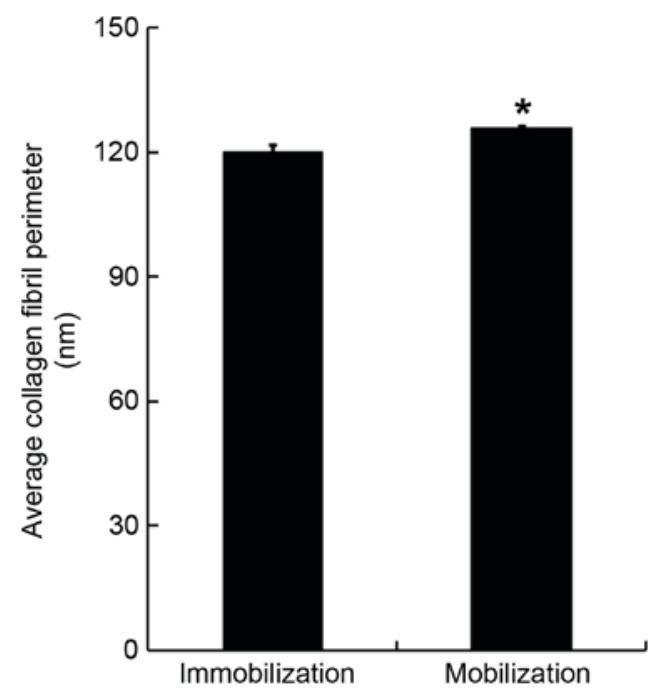

Figure 3. Mobilization increased the Achilles tendon collagen fibril perimeter. Average collagen fibril perimeter of the rats from the immobilization group $(n=13)$ and mobilization group $(n=12)$, as determined by scanning electron microscopy. ${ }^{\mathrm{P}}<0.05$.

increased fibroblast number and only moderate inflammatory cell infiltration (Fig. 1).

Ultrastructural examination. Differences in the ultrastructure of the Achilles tendon tissues between the immobilization group and the active mobilization group were compared using SEM. Similar to the results of histological staining, the findings revealed that Achilles tendon injury followed by immobilization resulted in disorganized collagen fibrils with varied gap size and thickness, with some collagen fibrils attached to each other, some fibril tangling, and fragmentation (Fig. 2). Conversely, the collagen fibrils in the mobilization group were tightly arranged with even thickness, and uniform intervals were detected between the collagen fibrils (Fig. 2). These findings suggested that mobilization therapy may prevent pathological alterations in the injured Achilles tendon by maintaining the normal structure and organization of collagen fibrils. The SEM images were also used to quantify the collagen fibril perimeter. It was demonstrated that the average collagen fibril perimeter in the

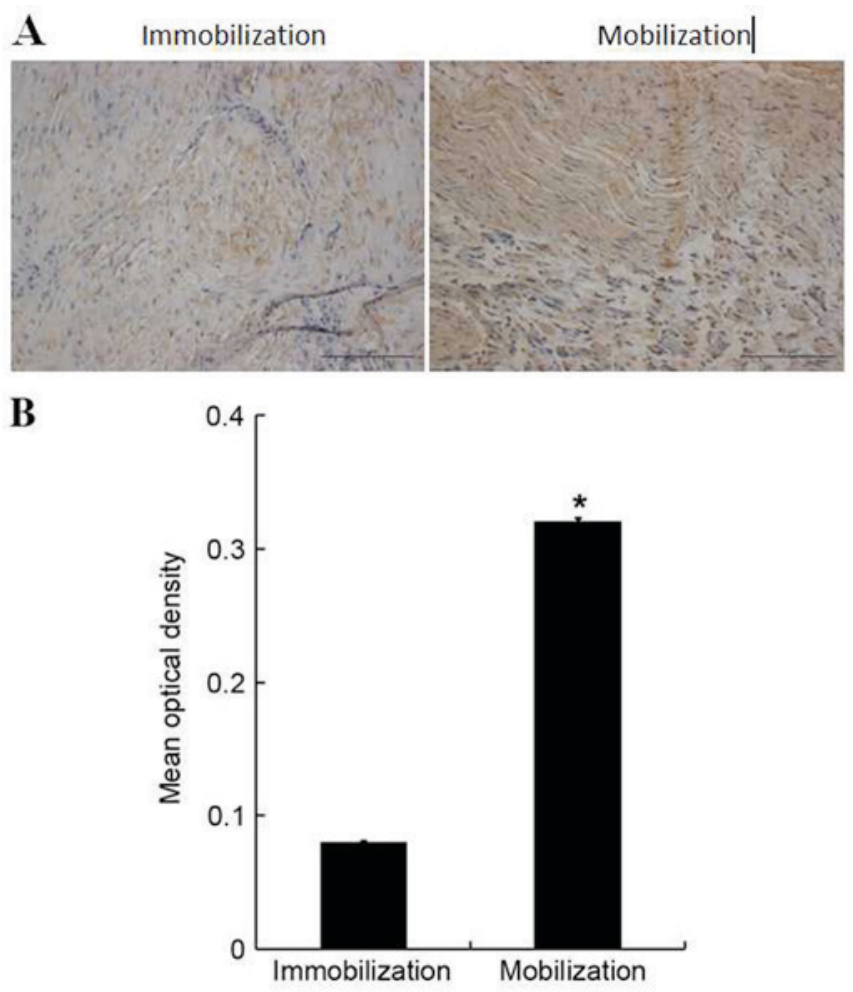

Figure 4. Immunohistological analysis of CRMP-2 expression in Achilles tendons. (A) Tissue sections derived from the mobilization group or immobilization group were immunostained with anti-CRMP-2 antibody. Magnification, x400; scale bar, $10 \mu \mathrm{m}$ (B) Mean optical density of CRMP-2 staining. ${ }^{*} \mathrm{P}<0.05$. CRMP-2, collapsin response mediator protein-2.

mobilization group was significantly increased compared with in the immobilization group $(125.6 \pm 0.8 \mathrm{~nm}$ vs. $119.9 \pm 1.7 \mathrm{~nm}$; $\mathrm{F}=6.075$, P $<0.05$; Fig. 3).

CRMP-2 expression. In order to determine the molecular mechanism underlying mobilization-mediated protection in rats with injured Achilles tendons, the expression of CRMP-2 was examined. Immunohistological analysis demonstrated a low CRMP-2 expression in the Achilles tendon tissues of immobilized animals (Fig. 4A). However, CRMP-2 expression was upregulated in the mobilization group, as evident 
$\mathbf{A}$
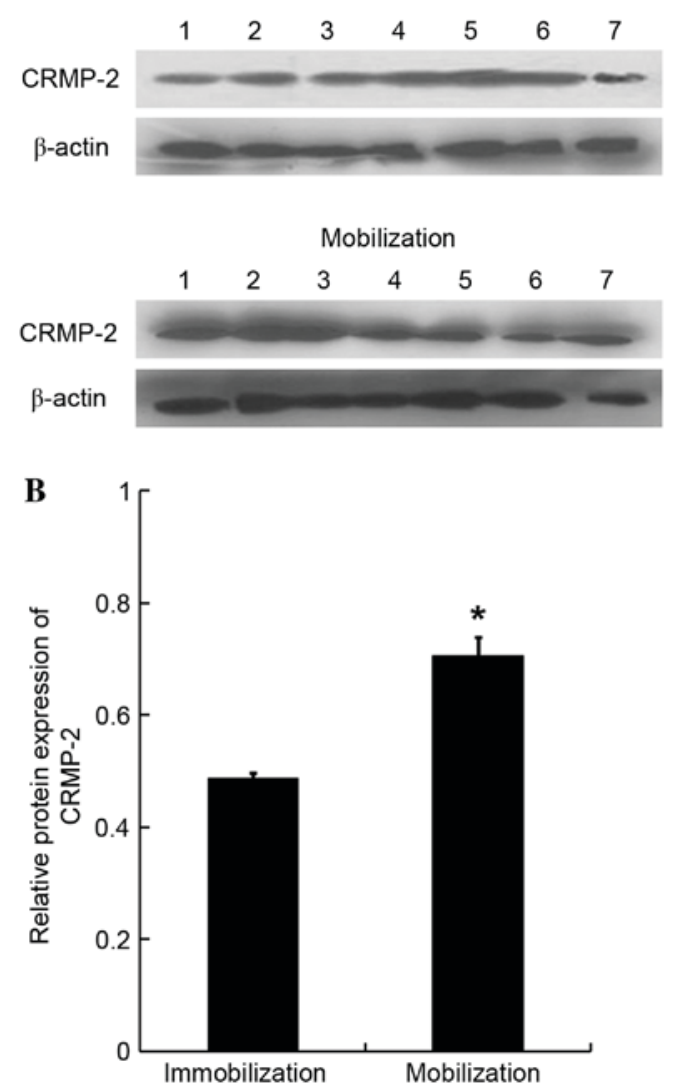

Figure 5.CRMP-2 protein expression analysis in Achilles tendons. (A) Tissues derived from mobilization $(n=7)$ and immobilization groups $(n=7)$ were used for immunoblotting with an antibody against CRMP-2. $\beta$-actin was used as an internal control. (B) Protein expression levels of CRMP-2 were normalized to $\beta$-actin. Data were calculated from 7 independent experiments. ${ }^{*} \mathrm{P}<0.05$. CRMP-2, collapsin response mediator protein-2.

by brown-yellow staining, particularly in the injured site of Achilles tendon tissues. Following quantification, it was determined that CRMP-2 expression was significantly increased in the Achilles tendon tissues of the mobilization group compared with the immobilization group $(0.32 \pm 0.00$ vs. $0.08 \pm 0.00 ; \mathrm{F}=1.332, \mathrm{P}<0.05$; Fig. 4B). Western blotting semi-quantification further confirmed that the protein expression levels of CRMP-2 were significantly upregulated in the Achilles tendon tissues of the mobilization group compared with the immobilization group $(0.71 \pm 0.03$ vs. $0.49 \pm 0.01 \mathrm{~nm}$; $\mathrm{F}=3.577, \mathrm{P}<0.05$; Fig. 5).

\section{Discussion}

The prevalence of Achilles tendon rupture in humans is relatively high, particularly in athletes. Compounding the problem is the fact that surgical outcomes are frequently poor due to various postoperative complications. It has previously been suggested that immobilization of the digit or limb may promote faster healing; however, this technique may eventually lead to the formation of adhesions between the tendon and tendon sheath, which may result in friction and reduced gliding (16). Therefore, the present study established a rodent model of Achilles tendon injury and demonstrated that active mobilization following Achilles tendon injury may promote healing by upregulation of CRMP-2 expression, particularly at the site of the injured Achilles tendon. In addition, mobilization through active exercise resulted in reduced pathological alterations in the injured Achilles tendon; rats in the mobilization group exhibited regular collagen fibril arrangement, increased collagen fibrils, elevated number of fibroblasts and moderate inflammatory cell infiltration. Consistent with the results of previous studies that identified a strong positive correlation between collagen fibril diameter and tendon strength $(8,17)$, the present study indicated that mobilization treatment significantly increased the mean collagen fibril perimeter. It is possible that these early adaptive responses may trigger long-term tendon structure remodeling and lead to changes in the tendon's mechanical properties $(14,18-20)$.

Various factors and mediators have been proposed to serve essential roles in the mechanobiological responses of cells, including collagens, proteoglycans and MMPs (19). In our previous study, protein expression was compared in rabbits from immobilization and active mobilization groups, and several differentially expressed proteins were identified in the rabbits receiving mobilization treatment, including myosin light chain 1, phosphoglycerate kinase, F-actin capping protein subunit $\alpha 1$, gil45478150-LRRGT00155 protein, prolyl 4-hydroxylase, $\alpha$ I subunit isoform 2 precursor and CRMP-2 (12). The present study detected increased CRMP-2 protein expression levels in rats with injured Achilles tendons that received mobilization treatment. The healing process consists of three phases: i) Inflammatory phase, ii) proliferative phase and iii) maturation phase. The increased CRMP-2 expression was particularly evident at the site of injury and it is possible that the increased CRMP-2 level occurred during the proliferative phase in injured animals that received mobilization treatment. A previous study demonstrated that active Achilles tendon kinesitherapy promoted neurite regeneration of the ruptured Achilles tendon (12); therefore, it is possible that active exercises promoted the Achilles tendon healing process by accelerating the local neurite regeneration and nerve repair mediated by CRMP-2 at the injury site. In addition, it has previously been reported that CRMP-2 accelerated axon regeneration of nerve-injured motor neurons in rats (21). These findings provide promising insights into tendon healing at the molecular level; however, the precise role of CRMP-2 in regulating nerve repair and Achilles tendon healing requires further investigation. These findings are consistent with those of Ackermann et al (22) and Bring et al (23), and are in accordance with the findings of our previous studies $(1,12,13,20,24,25)$.

In conclusion, the present study revealed that active mobilization therapy reduced Achilles tendon damage in a rodent model. Specifically, collagen fibril structure and arrangement was similar to that of healthy animals and was improved when compared with animals that did not undergo mobilization therapy. Notably, the protection of Achilles tendon injury achieved by active mobilization therapy may be associated with increased CRMP-2 protein expression, particularly at the site of injury. Future studies should focus on the underlying mechanism of CRMP-2-mediated Achilles tendon repair following acute injury using conditional knockout mice or 
cellular gene manipulation. The in situ expression of CRMP-2 should also be investigated in future clinical studies.

\section{Acknowledgements}

The present study was supported by the National Natural Science Foundation of China (grant no. 81460337).

\section{References}

1. Jielile J, Sabirhazi G, Chen J, Aldyarhan K, Zheyiken J, Zhao Q and Bai J: Novel surgical technique and early kinesiotherapy for acute Achilles tendon rupture. Foot Ankle Int 33: 1119-1127, 2012.

2. Arøen A, Helgø D, Granlund OG and Bahr R: Contralateral tendon rupture risk is increased in individuals with a previous Achilles tendon rupture. Scand J Med Sci Sports 14: 30-33, 2004

3. Khan RJ, Fick D, Keogh A, Crawford J, Brammar T and Parker M: Treatment of acute achilles tendon ruptures. A meta-analysis of randomized, controlled trials. J Bone Joint Surg Am 87: 2202-2210, 2005.

4. Hufner TM, Brandes DB, Thermann H, Richter M, Knobloch K and Krettek C: Long-term results after functional nonoperative treatment of achilles tendon rupture. Foot Ankle Int 27: 167-171, 2006.

5. Metz R, Kerkhoffs GM, Verleisdonk EJ and van der Heijden GJ: Acute Achilles tendon rupture: Minimally invasive surgery versus non operative treatment, with immediate full weight bearing. Design of a randomized controlled trial. BMC Musculoskelet Disord 8: 108, 2007.

6. Soroceanu A, Sidhwa F, Aarabi S, Kaufman A and Glazebrook M: Surgical versus nonsurgical treatment of acute Achilles tendon rupture: A meta-analysis of randomized trials. J Bone Joint Surg Am 94: 2136-2143, 2012.

7. Devries G: Surgical and nonsurgical treatment of achilles tendon rupture: The favorable effect of early functional rehabilitation. Clin J Sport Med 24: 159-160, 2014.

8. Battaglia TC, Clark RT, Chhabra A, Gaschen V, Hunziker EB and Mikic B: Ultrastructural determinants of murine achilles tendon strength during healing. Connect Tissue Res 44: 218-224, 2003.

9. Magnusson SP, Qvortrup K, Larsen JO, Rosager S, Hanson P, Aagaard P, Krogsgaard M and Kjaer M: Collagen fibril size and crimp morphology in ruptured and intact Achilles tendons. Matrix Biol 21: 369-377, 2002.

10. Karousou E, Ronga M, Vigetti D, Passi A and Maffulli N: Collagens, proteoglycans, MMP-2, MMP-9 and TIMPs in human achilles tendon rupture. Clin Orthop Relat Res 466: 1577-1582, 2008.

11. Yoshimura T, Kawano Y, Arimura N, Kawabata S, Kikuchi A and Kaibuchi K: GSK-3beta regulates phosphorylation of CRMP-2 and neuronal polarity. Cell 120: 137-149, 2005.
12. Jielile J, Aibai M, Sabirhazi G, Shawutali N, Tangkejie W, Badelhan A, Nuerduola Y, Satewalede T, Buranbai D, Hunapia B, et al: Active Achilles tendon kinesitherapy accelerates Achilles tendon repair by promoting neurite regeneration. Neural Regen Res 7: 2801-2810, 2012.

13. Jielile J, Bai JP, Sabirhazi G, Redat D, Yilihamu T, Xinlin B, Hu G, Tang B, Liang B and Sun Q: Factors influencing the tensile strength of repaired Achilles tendon: A biomechanical experiment study. Clin Biomech (Bristol, Avon) 25: 789-795, 2010.

14. Tang B, Bai JP and Jielile J: The histological effects of early mobilization in the healing after Achilles tendon of rabbits rupture repair. J Chin Physicians 12: 19-22, 2010.

15. Jacob KM and Paterson R: Surgical repair followed by functional rehabilitation for acute and chronic achilles tendon injuries: Excellent functional results, patient satisfaction and no reruptures. ANZ J Surg 77: 287-291, 2007.

16. James R, Kesturu G, Balian G and Chhabra AB: Tendon: Biology, biomechanics, repair, growth factors, and evolving treatment options. J Hand Surg Am 33: 102-112, 2008.

17. Derwin KA and Soslowsky LJ: A quantitative investigation of structure-function relationships in a tendon fascicle model. J Biomech Eng 121: 598-604, 1999.

18. Wang JH: Mechanobiology of tendon. J Biomech 39: 1563-1582, 2006.

19. Wang JH and Thampatty BP: An introductory review of cell mechanobiology. Biomech Model Mechanobiol 5: 1-16, 2006.

20. Jiasharete J and Bai JP: Mechanobiology during Achilles tendon healing. Chin J Clin Rehabil Tissue Eng Res 11: 17-20, 2008.

21. Suzuki Y, Nakagomi S, Namikawa K, Kiryu-Seo S, Inagaki N, Kaibuchi K, Aizawa H, Kikuchi K and Kiyama H: Collapsin response mediator protein-2 accelerates axon regeneration of nerve-injured motor neurons of rat. J Neurochem 86: 1042-1050, 2003.

22. Ackermann PW, Ahmed M and Kreicbergs A: Early nerve regeneration after achilles tendon rupture-a prerequisite for healing? A study in the rat. J Orthop Res 20: 849-856, 2002.

23. Bring DK, Kreicbergs A, Renstrom PA and Ackermann PW: Physical activity modulates nerve plasticity and stimulates repair after Achilles tendon rupture. J Orthop Res 25: 164-172, 2007.

24. Jielile J, Badalihan A, Qianman B, Satewalede T, Wuerliebieke J, Kelamu M and Jialihasi A: Clinical outcome of exercise therapy and early post-operative rehabilitation for treatment of neglected Achilles tendon rupture: A randomized study. Knee Surg Sports Traumatol Arthrosc 24: 2148-2155: 2016.

25. Badalihan A, Aihemaiti A, Shawutali N, Jielile J, Jialihasi A, Tangkejie W, Nuerdoula Y, Satewalede T, Hunapiya B, Niyazebieke $\mathrm{H}$, et al: Outcome of a one-stage tensile stress surgical technique and early postoperative rehabilitation in the treatment of neglected achilles tendon rupture. J Foot Ankle Surg 54: 153-159, 2015. 